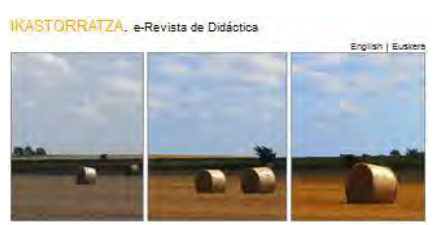

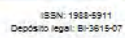

Inicio, Sobre nosotros, Publicacionesl, Participa

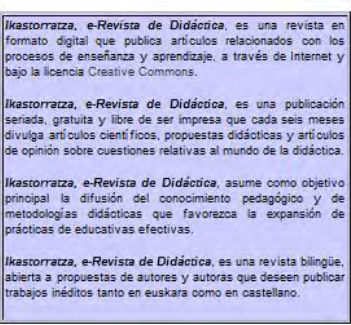

IKASTORRATZA. Didaktikarako e-aldizkaria

IKASTORRATZA. e-journal on Didactics

ISSN: 1988-5911 (Online) Journal homepage: http://www.ehu.eus/ikastorratza/

\title{
El aprendizaje de las plantas como seres vivos: una metodología basada en el dibujo infantil
}

Xabier Villanueva

Universidad del País Vasco/Euskal Herriko Unibertsitatea

(UPV/EHU)

xvillanueva001@ikasle.ehu.eus

To cite this article:

Villanueva, X. (2017) El aprendizaje de las plantas como seres vivos: una metodología basada en el dibujo infantil. IKASTORRATZA. e-Revista de Didáctica, 18, 106-123. DOI: $10.37261 / 18$ alea/6

To link to this article:

http://www.ehu.es/ikastorratza/18_alea/6.pdf

Published online: 20 Jun 2017. 


\title{
El aprendizaje de las plantas como seres vivos: una metodología basada en el dibujo infantil
}

Xabier Villanueva

Escuela Universitaria de Magisterio de Bilbao Universidad del País Vasco/Euskal Herriko Unibertsitatea (UPV/EHU) xvillanueva001@ikasle.ehu.eus

\section{Resumen}

Estudios previos han investigado el desarrollo del concepto de ser vivo en edades tempranas desde diferentes perspectivas metodológicas. Sin embargo, estos enfoques ponen excesivo énfasis en la comunicación verbal a la hora de llevar a cabo las tareas lo que puede llevar a limitaciones a la hora de medir este concepto biológico. Por ello, se presenta una aproximación desde una metodología no verbal basada en las producciones pictóricas del alumnado. La presente investigación tiene como objetivo analizar la relación entre la noción de ser vivo y el dibujo infantil en una muestra de 197 niños y niñas de entre 4 y 8 años de edad. Para ello, se realizan tareas de categorización de imágenes de seres vivos e inertes y la recogida de expresiones gráficas sobre el mundo vegetal. Los resultados de las tareas realizadas apuntan la consideración de que el alumnado con el conocimiento más preciso sobre si las plantas están vivas dibujan más frecuentemente elementos pictóricos relacionados con la vida como el sol, el agua, semillas, las nubes, y tienden a dibujar también más tipos de plantas.

Palabras clave: Desarrollo conceptual, concepto ser vivo, plantas, producciones pictóricas.

\begin{abstract}
Previous studies have investigated the development of the concept of being alive at an early age from different methodological perspectives. However, these approaches place excessive emphasis on verbal communication which can lead to limitations when it comes
\end{abstract}


to measure this biological concept. Therefore, a methodology based on the pictorial productions of students is presented. The present research aims to analyze the relationship between the notion of being alive and children 's drawings in a sample of 197 boys and girls of 4-8 years old. For this, a categorization task is performed with images of living and inert beings, followed by the collection of graphic expressions about the plant world. The results point to the fact that students with the most precise knowledge about plants are alive draw more frequently pictorial elements related to life such as sun, water, seeds, clouds and tend to draw more plants types.

Key words: Conceptual development, Living thing concept, plants, drawings.

\section{Introducción}

Comprender qué elementos de nuestro alrededor están vivos, y cuáles no, es una competencia básica que se construye durante la infancia mediante distintas exposiciones y experiencias con el contexto natural, social y cultural del alumnado. Además, es una conceptualización biológica básica que se trabaja en el currículo de educación infantil y primaria.

A continuación, se presenta un estudio sobre el desarrollo del concepto de ser vivo de las plantas en niños y niñas de edades comprendidas entre los 4 y 8 años. Con el propósito de analizar el marco de investigación que nos ocupa, la revisión teórica se centra en dos áreas diferentes de investigación. Por un lado, los estudios centrados en la comprensión del concepto de ser vivo y su desarrollo en la edad infantil. Por otro lado, se presentan los dibujos infantiles como instrumento eficaz para inferir el pensamiento y la simbología del alumnado.

\subsection{Desarrollo del concepto de ser vivo}

Desde los estudios clásicos piagetianos (Piaget, 1929) se ha intentado investigar la comprensión del alumnado más pequeño a la hora distinguir entre lo que está vivo y no está vivo, específicamente, desde dos vertientes teóricas y metodológicas diferenciadas. Por un lado, la perspectiva neopiagetiana que da continuidad al carácter cualitativo del estudio piagetiano enfatizando las limitaciones del alumnado al representar relaciones lógicas subyacentes a fenómenos biológicos. Niños y niñas parten de una psicología ingenua sin un marco conceptual y psicológico maduro, y no son capaces hasta cierta etapa evolutiva de realizar una correcta representación biológica de lo vivo (Carey, 
1985). Por otro lado, desde la perspectiva postpiagetiana se defiende el desarrollo de una elaboración mental progresiva del concepto de ser vivo en el que se parte de una competencia ya existente, con un amplio alcance, pero limitado en precisión y detalle (Erickson et al., 2010).

En las últimas décadas, ha surgido un amplio consenso a favor del enfoque postpiagetiano (Inagaki y Hatano, 2006). Esto puede deberse a que el desempeño limitado exhibido en tareas de categorización por niños y niñas puede no deberse a una insuficiencia conceptual, sino a problemas al asociar el término con su conceptualización (Medin y Atran, 2004), ya sea por el todavía débil desarrollo del lenguaje o por la capacidad de realizar abstracciones. Estudios recientes han empleado una metodología de preguntas iniciada por los niños y niñas para evaluar el concepto de ser vivo, procedimiento que no requiere que el alumnado se adapte a la terminología del investigador. Estos estudios sugieren una mejor comprensión por parte de alumnado de edad infantil (Kemler Nelson, Egan y Holt, 2001).

Dentro de esta línea de investigación se ha analizado la comprensión conceptual de lo que está vivo y no está vivo desde diferentes perspectivas como pueden ser el enfoque neuropsicológico, la perspectiva funcional, la fundamentación teleológica o el estudio de la animacidad. A continuación, se presenta cada una de estas aproximaciones y sus principales contribuciones.

Desde un punto de vista neuropsicológico se considera que la capacidad de clasificar entidades vivas e inertes surge en los primeros meses de vida (Molina, Van de Walle, Condry y Spelke, 2004). Estos estudios ponen en evidencia una estructura cognitiva innata para este concepto (Spelke y Kinzler, 2007) que ha venido desarrollándose evolutivamente siendo vital para la supervivencia del individuo en los primeros años de vida.

La perspectiva funcional investiga la comprensión del concepto de ser vivo en niños y niñas mediante la distinción clara entre lo vivo y no vivo con respecto a las propiedades biológicas. Entre las variables más estudiadas encontramos características como morir, crecer o brotar (Greif et al., 2006; Waxman, 2005). Se ha encontrado que el alumnado más pequeño atribuye la necesidad de alimento y muerte tanto a plantas como a animales (Inagaki y Hatano 2002). 
Otra dimensión importante que se ha venido estudiando es la referente a la interpretación de los fenómenos biológicos sobre la base de sus propósitos, fines y funciones para las que se han creado (Casler y Kelemen, 2007). En esta perspectiva teleológica se ha percibido que niños y niñas de tan solo 3 años de edad daban información biológica al encontrarse con animales nuevos y valor funcional, en cambio, al encontrarse con artefactos nuevos (Greif et al. 2006; Kemler Nelson et al., 2001).

Varios autores han focalizado esta comprensión desde la capacidad de movimiento que infieren niños y niñas de cada elemento en cuestión, esté vivo o no vivo. Niños y niñas esperan que las cosas estén vivas para moverse (Carey, 1985) y rara vez clasifican a los elementos inmóviles como seres vivos (Richard y Siegler, 1984). Cuando se les pide que argumenten o justifiquen por qué algo está vivo a menudo se refieren a la capacidad de movimiento de la entidad (Gelman y Kremer, 1991). Sin embargo, esta comprensión no se traduce en una integración fiable a la hora de categorizar o etiquetar a las plantas como seres vivos (Meunier y Cordier, 2004; Richard y Siegler, 1984).

Finalmente, una última línea de investigación ha intentado focalizar la comprensión de ser vivo desde una perspectiva esencialmente teleológica. Según ésta, el alumnado interpreta los fenómenos naturales sobre la base de los fines, propósitos o funciones para los que se han creado (Casler y Kelemen, 2007; Kelemen y Rosset, 2005). Cabe destacar que esta no es una característica exclusiva del pensamiento infantil ya que se da también muy frecuentemente en adultos en una amplia gama de fenómenos biológicos y geológicos (Kelemen y Rosset, 2009).

Autores como Yorek, Sahin y Aydin (2009) han sugerido un desarrollo progresivo del concepto ser vivo, en el que se le concede en primer lugar al ser humano, después a los animales y finalmente a las plantas. En suma, la mayoría de la literatura mencionada coincide en remarcar el conocimiento limitado que el alumnado muestra acerca de la vida de las plantas cuando se intenta atribuir el estado de vida a estas (Gatt, Tunnicliffe, Borg y Lautier, 2007; Leddon et al., 2009; Opfer y Siegler, 2004).

\subsection{El dibujo infantil sobre la naturaleza}

La mayoría de investigaciones relativas al desarrollo del concepto de ser vivo y razonamiento normativo medioambiental en la infancia se basan en el análisis de la expresión oral como metodología para estudiar los pensamientos del alumnado. Sin embargo, existen otros procedimientos alternativos para explorar las ideas que tienen 
niños y niñas sobre este tema (Rollins, 2005). El dibujo es una técnica muy valiosa para estudiar el pensamiento infantil, sobre todo, en alumnado con dificultades para expresarse oralmente (Holliday, Harrison y McLeod, 2009).

Autores como Rennie y Javis (1995) ven la metodología mediante dibujos más adecuada para reflejar los pensamientos del alumnado. Esto puede ser debido al hecho de tener que dar respuestas que coincidan con las respuestas convencionales. El dibujo se ve como un espejo en el que se manifiesta la propia evolución simbólica de la mente (Cherney, Seiwert, Dickey y Flichtbeil, 2006) y un reflejo del mundo interior del alumnado (Malchiodi, 1998; Salmon y Lucas, 2011). Además, los estudios basados en estos procedimientos pictóricos también se han considerado eficaces a la hora de llevar a cabo investigaciones interculturales (Michael y Rajuan, 2009).

El dibujo infantil es un procedimiento metodológico utilizado con bastante éxito en estudios que quieren analizar la comprensión temprana de niños y niñas. Estos diseños de investigación han analizado como niños y niñas comprenden una amplia gama de conceptos: los bosques y sus habitantes (Snaddon, Turner y Foster, 2008), las plantas (Villarroel, 2016), células de las plantas (Topsakal y Oversby, 2012), el cuerpo humano (Prokop y Fancovicová, 2006), la tierra y universo (Hannust y Kikas, 2007), conceptos geológicos (Shepardson, Wee, Priddy y Harbor, 2007) y sobre las concepciones tecnológicas (Rennie y Jarvis 1995).

El uso de esta metodología, sin embargo, trae consigo ciertas dificultades como es su complicada cuantificación (Muijs, 2004). Aun así, varios trabajos previos proponen una metodología para el estudio de elaboraciones pictóricas en escolares de entre 4 y 7 años sobre las plantas y fenómenos atmosféricos (Dove 2011; Tunnicliffe 2004; Tunnicliffe y Reiss 2001; Villarroel, 2016).

Una línea de trabajo ciertamente interesante ha intentado relacionar la comprensión del concepto de ser vivo del alumnado y los elementos pictóricos representados en sus dibujos (Villarroel e Infante, 2014), encontrando una estrecha relación entre ambas.

\subsection{Objetivos de la investigación}

La investigación realizada hasta el momento deriva de un extenso cuerpo de estudios con una minuciosa precisión metodológica, sin embargo, pone demasiado énfasis en la capacidad y desarrollo lingüístico del alumnado, sin tener en cuenta su posible 
influencia a la hora de conceptualizar lo que está vivo y no vivo (véase, Leddon et al, 2009). Pocos estudios han empleado una clase amplia de metodología no verbal para investigar la comprensión de los seres vivos.

Por ello, se presenta la siguiente investigación con el objetivo de examinar la competencia a la hora de diferenciar entre seres vivos e inertes de niñas y niños de edad infantil, relacionando este aspecto con el contenido dibujado en sus producciones pictóricas. Para lograr esta meta se proponen los siguientes dos objetivos específicos:

1- Conocer como el alumnado de la última etapa de Educación Infantil y primeros años de educación Primaria diferencian seres vivos y entidades inertes a través de una tarea de categorización con el fin de valorar su comprensión relativa al concepto de ser vivo.

2- Analizar los dibujos que los niños y niñas llevan a cabo en torno al mundo vegetal para estudiar su comprensión sobre las plantas.

La investigación tiene como propósito final aportar más evidencias a un marco de investigación que intenta analizar cómo se desarrolla la conceptualización de ser vivo en la etapa infantil y primaria. Sin embargo, este estudio también está ligado al interés de facilitar evidencias para la creación y puesta en marcha de intervenciones educativas más eficaces en el ámbito del aprendizaje de las ciencias naturales.

\section{Metodología}

\subsection{Aspectos éticos}

La metodología de investigación es presentada a la dirección académica y el profesorado de los centros educativos que han participado en el presente estudio. Posteriormente, es aprobada con el consiguiente consentimiento paterno o materno de todo el alumnado que participó en ella. El estudio se lleva a cabo en los últimos dos meses del año 2015 y en el primer mes del año 2016, en dos diferentes colegios de la comarca del Gran Bilbao, en la provincia de Bizkaia, España.

\subsection{Participantes}

En la presente investigación participan un total de 197 niños y niñas de edades comprendidas entre los 4 y 8 años edad, es decir, alumnado que cursa actualmente los dos últimos cursos de Educación Infantil y los dos primeros cursos de la etapa de Educación Primaria. Un 46,7\% de la muestra seleccionada son niños, mientras que el 53,3\% son 
niñas. En cuanto al nivel educativo, un $22,3 \%$ de la muestra participante pertenece a los 4 años de Educación Infantil, un 25,4\% a los 5 años de Educación Infantil, un 23,9\% al primer curso de Educación Primaria (6-7 años) y un 28,4\% al segundo curso de Educación Primaria (7-8 años).

\subsection{Entrevista}

La metodología propuesta para llevar a cabo los objetivos de investigación se fundamenta en entrevistas con el alumnado, de manera individual. Todas estas entrevistas individuales están dirigidas por el mismo investigador y tienen una duración aproximada de unos 20 minutos.

Antes de comenzar las entrevistas con el alumnado participante se pone en marcha una actividad de contacto inicial con el aula habitual y con todo el grupo. Como norma a seguir, el profesorado introduce al investigador al alumnado y, posteriormente, se pasa a explicar el procedimiento que se va a llevar a cabo en los siguientes días. Las entrevistas se suelen llevar a cabo en la misma aula en un lugar un poco apartado, o en su caso, en otro espacio fuera del aula, pero no muy alejado de la misma para que el alumnado no se sienta desplazado.

El propósito de esta actividad grupal es motivar al alumnado hacia las tareas de investigación que se van a llevar a cabo individualmente. Con este objetivo se cuenta una historieta mediante la ayuda de una marioneta, explicando que esta viene de un desierto lejano donde no hay plantas y necesita su ayuda para aprender sobre el mundo vegetal. El investigador termina la historia pidiendo al alumnado su apoyo contándole a la marioneta todo lo que saben sobre las plantas en las siguientes dos tareas de investigación que se van a realizar. Estas dos tareas, siguiendo el orden en el que aparecen, son The Living/non-living Distinction Test y un test de dibujo libre. La descripción de cada tarea y su respectivo procedimiento se presenta a continuación.

La primera tarea, siguiendo la propuesta de Villarroel (2013) y Leddon et al. (2009), se trata de un test de categorización mediante imágenes llamado The Living/nonliving Distinction Test que tiene como propósito analizar la comprensión que tienen niños y niñas para diferenciar seres vivos de otras entidades no vivas.

Los participantes deben categorizar diferentes entidades como seres vivos o inertes. Para conseguir este objetivo, al alumnado participante en la investigación se le presenta individualmente ocho fotografías a color de tamaño $18 \times 10 \mathrm{~cm}$. Estas fotografías, 
seleccionadas previamente, muestran cuatro seres vivos, entre las que se encuentran una planta, un árbol, un perro y un pájaro, y cuatro entidades no vivas, tales como un coche, una moto, un sol y unas nubes. Estas entidades se dividen en 4 categorías: Animales (perro y pájaro), Vehículos (coche y moto), Vegetal (planta y árbol) y Fenómenos atmosféricos (sol y nubes).

Para ello, se les pregunta, en cada caso, si la entidad mostrada está viva mediante el uso de la siguiente estructura interrogativa: “¿Está X vivo?”. Su traducción al euskera sería: "X biziririk dago?”. El investigador recoge las respuestas de cada entidad gráfica mostrada tomando nota de los aciertos y de los errores realizado por cada niño y niña participante.

El criterio empleado que se basa para clasificar las respuestas del alumnado como acertadas es la correcta clasificación de las dos entidades de cada categoría. De esta manera un solo error en alguna de las dos entidades presentadas llevaría a la clasificación incorrecta de la categoría.

La segunda tarea consiste en un test de dibujo libre. En esta tarea se les pide a los niños y niñas que pinten un dibujo sobre plantas. El alumnado tiene a su disposición hojas de papel, bolígrafos y pinturas de colores específicos para poder realizar la ilustración. Una vez que el autor o autora exprese que ha finalizado el dibujo, el entrevistador procede a revisar con él el significado de todos los elementos dibujados en la ilustración.

\subsection{Análisis estadístico}

Las variables que se van a considerar en la presente investigación son las siguientes:

- Sexo, edad y curso académico del alumnado.

- Si las entidades del test The Living/Non-living Distinction Test se han clasificado correctamente o no. Es una variable categórica dicotómica que toma dos posibles valores (correcto o incorrecto).

- El número de elementos pictóricos dibujados relacionados con el mundo vegetal, sin relación con el mundo vegetal, así como el total de elementos dibujados.

Con respecto a los procedimientos estadísticos, la asociación entre las variables dicotómicas es analizada mediante el análisis Chi-cuadrado con el correspondiente examen del tamaño del efecto mediante la V de Cramer (Kline 2004). 
Para analizar la relación entre las variables discretas obtenidas en el test de dibujo libre se utiliza las pruebas no-paramétricas U de Mann-Whitney para la variable sexo y H de Kruskal-Wallis para la variable edad (Siegel y Castellan, 1988). Se usa una prueba no paramétrica ya que las variables discretas configuradas no son consistentes con una distribución normal. El tamaño del efecto fue calculado mediante el parámetro r y Etacuadrado ( $\eta 2$ ), que se interpretaran según los criterios de Morse (1999) y Prajapati, Dunne y Armstrong (2010).

El nivel de significación utilizado en los diferentes análisis que se realizan durante toda la investigación es de 0,05 y el software utilizado para estos fue la versión 20 del SPSS.

\section{Resultados}

En este apartado se presenta el análisis de datos realizado en dos partes diferenciadas con relación a las variables sexo y curso académico del alumnado. En primer lugar, se comenzará con el análisis del test Living/non-living Distinction Test y, a continuación, se detallarán los resultados referentes al test de dibujo libre.

\subsection{The Living/non-living Distinction Test}

A la hora analizar los datos obtenidos en la realización de la tarea The Living/nonliving Distinction Test, por parte de toda la muestra participante, se constata que ningún estudiante de 4 años y 5 años es capaz de clasificar correctamente todas las entidades que se presentan en las 8 fotografías. En cambio, solo un 19,1\% del alumnado de 6-7 años consigue clasificar correctamente todas las entidades, mientras que un $41,1 \%$ de los niños y niñas entre 7-8 años resuelven correctamente el test. En la siguiente Tabla 1 aparecen las frecuencias de respuestas correctas alcanzadas por cada grupo de edad en las cuatro categorías de identidades.

Si se atiende a la variación de las frecuencias observadas entre los cuatro niveles educativos se encuentran diferencias estadísticamente significativas en las categorías Vehículos (Chi-cuadrado $=17,92$ (3); $\mathrm{p}<0,001 ; \mathrm{V}$ de Cramer $=0,32$ ), Fenómenos atmosférica (Chi-cuadrado $=12,48(3) ; \mathrm{p}<0,01$; V de Cramer $=0,32)$ y Animal (Chicuadrado $=20,98(3) ; \mathrm{p}<0,001 ; \mathrm{V}$ de Cramer $=0,32)$. El tamaño de efecto indicado mediante el parámetro $\mathrm{V}$ de Cramer permite suponer no menos que una relación media entre las variables acierto a la hora de categorizar las entidades y el curso educativo del 
alumnado. Sin embargo, no se han encontrado diferencias estadísticamente significativas a la hora de analizar la categoría Vegetal (flores y árbol).

Tabla 1. Frecuencias relativas (\%) de cada uno de los elementos pictóricos encontrados en los dibujos de la muestra $(\mathrm{N}=197)$

\begin{tabular}{lcccc}
\hline \multicolumn{1}{c}{ Categorías } & Animal & $\begin{array}{c}\text { Fenómenos } \\
\text { Atmosféricos }\end{array}$ & Vegetal & Vehículos \\
\hline 4 años $(\mathrm{N}=44)$ & 75 & 47,7 & 43,2 & 47,7 \\
5 años $(\mathrm{N}=50)$ & 94 & 26 & 62 & 40 \\
$1^{\text {o }}$ Primaria & 93,6 & 55,3 & 55,3 & 66 \\
$2^{\circ}$ Primaria & 100 & 57,1 & 67,9 & 76,8 \\
\hline
\end{tabular}

En relación a la variable sexo, no se observa la existencia de diferencias estadísticamente significativas entre niños y niñas en cuanto a la realización del test The Living/non-living Distinction Test. Esta tendencia en los resultados se da tanto al considerar el conjunto de respuestas correctas como con relación a cada categoría.

\subsection{Test de dibujo libre}

Los resultados relativos al estudio de los dibujos llevados a cabo por el alumnado se presentan atendiendo a los datos relacionados con el análisis del contenido de dibujos. La Tabla 2 presenta la lista de todos los elementos pictóricos encontrados en el análisis de los dibujos, así como la frecuencia relativa de cada uno de estos elementos.

Como se detalla en la tabla 1 , los elementos pictóricos que se encuentran en los dibujos de la muestra estudiada pertenecen a los siguientes temas:

- Elementos relacionados con el mundo de las plantas y la diversidad vegetal: flores, árboles, verduras, otro tipo de órganos relacionados con la anatomía vegetal (hojas, raíces, frutos y semillas), animales salvajes, insectos, hongos, elementos contextuales que apoyan la escena representada en los dibujos (la tierra, el cielo y las montañas) y agentes atmosféricos como el sol, las nubes, la lluvia y arcoíris.

- Otros elementos relacionados no relacionadas directamente con la vida vegetal, pero si con la vida humana diaria como infraestructuras, vehículos, personas y también elementos decorativos tales como corazones. 
Tabla 2. Frecuencias relativas (\%) de cada uno de los elementos pictóricos encontrados en los dibujos de la muestra $(\mathrm{N}=197)$

\begin{tabular}{|c|c|c|c|}
\hline Categoría Mayor & Categoría Menor & \multicolumn{2}{|c|}{ Elementos Pictóricos } \\
\hline \multirow{9}{*}{$\begin{array}{c}\text { Elementos } \\
\text { Pictóricos } \\
\text { relacionados con } \\
\text { plantas }\end{array}$} & \multirow{4}{*}{ Plantas } & Flor: 82,7 & Hierba: 25,8 \\
\hline & & Árbol: 27,9 & Hoja: 48,7 \\
\hline & & Semilla: 1,5 & Raíz: 6 \\
\hline & & Planta: 15,7 & Fruto: 10,6 \\
\hline & Otros seres vivos & Vertebrado: 8,4 & Insecto: 10,5 \\
\hline & \multirow{2}{*}{$\begin{array}{l}\text { Elementos de } \\
\text { fondo }\end{array}$} & Suelo: 31,4 & Cielo: 23,8 \\
\hline & & Montaña: 4,2 & \\
\hline & \multirow{2}{*}{$\begin{array}{c}\text { Agentes } \\
\text { atmosféricos }\end{array}$} & Sol: 28,4 & Nube: 19,2 \\
\hline & & Agua: 6,6 & Arcoíris: 1,5 \\
\hline \multirow{2}{*}{$\begin{array}{l}\text { Otros elementos } \\
\text { pictóricos }\end{array}$} & \multirow{2}{*}{ Otros } & Casa: 6 & Vehículo: 2,1 \\
\hline & & Corazones: 2,5 & Personas: 10,6 \\
\hline
\end{tabular}

En la siguiente Tabla 3 se presentan los descriptores estadísticos de los elementos pictóricos relacionados con las plantas, los elementos pictóricos que no están relacionadas directamente con las plantas y el número total de elementos pictóricos dibujados. Estos elementos se clasifican en los 4 cursos educativos a los que pertenece el alumnado.

Tabla 3. Frecuencias relativas (\%) de cada uno de los elementos pictóricos encontrados en los dibujos de la muestra $(\mathrm{N}=197)$

\begin{tabular}{ccccc}
\hline Curso educativo & $\begin{array}{c}\text { Elementos } \\
\text { relacionados con } \\
\text { plantas }\end{array}$ & $\begin{array}{c}\text { Elementos sin } \\
\text { relación con } \\
\text { plantas }\end{array}$ & $\begin{array}{c}\text { Número total de } \\
\text { elementos } \\
\text { dibujados }\end{array}$ \\
\hline 4 años & Mediana & 2 & 0 & 2 \\
$\mathrm{~N}=44$ & Media (SD) & $2,09(1,5)$ & $0,45(0,7)$ & $2,55(2)$ \\
5 años & Mediana & 2 & 1 & 3 \\
$\mathrm{~N}=50$ & Media (SD) & $2,78(1,8)$ & $0,78(0,9)$ & $3,56(2,2)$ \\
$1^{\text {a }}$ Primaria & Mediana & 3 & 0 & 3 \\
$\mathrm{~N}=47$ & Media (SD) & $3,32(1,8)$ & $0,38(0,5)$ & $3,7(2)$ \\
$2^{\text {a }}$ Primaria & Mediana & 5 & 0 & 5 \\
$\mathrm{~N}=56$ & Media (SD) & $5,05(2,6)$ & $0,29(0,5)$ & $5,34(2,7)$ \\
\hline
\end{tabular}


Existen diferencias estadísticamente significativas en la variación de elementos pictóricos relacionados con las plantas (Kruskal-Wallis H-test $=44,51(3) ; p<0.01 ; \eta 2=0.22$ ), como en el número total de elementos dibujados (Kruskal-Wallis H-test =33,16 (3); p < $0.001 ; \eta 2=0.19)$ y el número de elementos pictóricos sin relación (Kruskal-Wallis Htest $=11,78(3) ; \mathrm{p}<0.01 ; \eta 2=0.06)$ en los diferentes cursos educativos.

Con relación a la variable sexo, no existen diferencias estadísticamente significativas en el número de elementos pictóricos dibujados que tienen vínculo con las plantas y ni tampoco número total de elementos pictóricos dibujados. En cambio, sí existen diferencias estadísticamente significativas en la variabilidad de los elementos pictóricos sin relación directa con las plantas entre los 4 cursos educativos $(z=-2,091$ (1); $\mathrm{p}<0.05 ; \mathrm{r}=0,14)$. Con todo, las conclusiones deben ser tomadas con prudencia ya que el tamaño del efecto sugiere una baja relación entre las variables consideradas.

\section{Discusión de resultados}

A la hora de distinguir entre seres vivos e inertes, ningún niño o niña de edades comprendidas entre los 4 y 5 años es capaz de diferenciar correctamente todas las distintas entidades vivas y no vivas presentadas. Esta tendencia encontrada coincide con otras investigaciones previas en las que se pone de relieve la falta de comprensión de este concepto biológico en edades tempranas (Carey, 1985; Inagaki y Hatano, 2013; Slaughter et al. 1999; Villarroel, 2013).

Esta limitación conceptual mostrada por los participantes más jóvenes puede deberse entre otros motivos a la tendencia incorrecta de incluir entidades móviles, como pueden ser las nubes, los coches, el sol o las motas, en la categoría de ser vivo (efecto de animacidad). Por el contrario, es interesante observar que niños y niñas de esta etapa de infantil son incapaces de categorizar las plantas como seres vivos dada su naturaleza aparentemente estática. Estos resultados coinciden con línea mostrada en otras investigaciones anteriores que enfatizan esta falta de comprensión entorno a las plantas y al mundo vegetal (Anggoro, Waxman y Medin, 2005; Gatt et al. 2007; Leddon et al., 2009; Opfer y Siegler, 2004).

Esta tendencia, en cambio, se va invirtiendo en los primeros años de la etapa de educación primaria (6-8 años) mejorando sustancialmente en cuanto a la comprensión y categorización de entidades vivas como inertes. En especial, el alumnado de estas edades 
atribuye con más frecuencia la característica biológica de vida a las plantas, mientras que clasifican a los medios de transporte y los fenómenos atmosféricos como objetos inertes.

Esta mejora sustancial a la hora de comprender la conceptualización de ser vivo puede deberse a diferentes causas ambientales y cognitivas. Varios autores han explicado esta tendencia mediante el aumento de conocimiento, experiencias y desarrollo de la capacidad cognitiva del alumnado entorno a esta noción biológica (Solomon y Zaitchik, 2012; Villarroel, 2013; Yorek, Sahin y Aydin, 2009).

En cuanto a la variable sexo, sin embargo, parece no existir una relación entre la comprensión de lo que está vivo y no está vivo y la variable sexo. Estas conclusiones van en línea con resultados obtenidos en estudios previos realizados por Villarroel (2013).

Respecto a las producciones pictóricas, los datos obtenidos muestran que el alumnado despliega una amplia variedad de elementos pictóricos relacionados con las plantas. Estos elementos pictóricos van desde distintos tipos de plantas (flores, hierba, árboles, arbustos) hasta partes detalladas de la morfología de las plantas (hojas, semillas, raíces, frutos) pasando por factores críticos que tienen relación e influyen en la vida vegetal (sol, agua, lluvia, tierra, nubes, insectos). También se representan otros seres vivos (personas, vertebrados) y elementos contextuales que suelen aparecer en muchos dibujos sobre otras temáticas (coches, corazones, casas).

Varios estudios anteriores señalan la utilización de elementos pictóricos similares en los dibujos relacionados con el mundo vegetal (Bowker 2007; Dove 2011; Tunnicliffe 2004; Tunnicliffe y Reiss 2001). De hecho, se puede constatar que se encuentran porcentajes y una evolución parecida en los resultados obtenidos por Villarroel e Infante (2014) a la hora de dibujar diferentes elementos.

Según se avanza en la edad se ve un crecimiento en la cantidad de elementos pictóricos dibujados relacionados con las plantas. Este aumento va relacionado con la capacidad de niños y niñas de ir comprendiendo las plantas como seres vivos, ya que los relacionan con elementos vitales como el agua, la lluvia, el sol y la tierra.

Los resultados obtenidos en las dos pruebas realizadas, tanto los resultados del test Living/non-Living Test como los del test de dibujo libre, sugieren que el desarrollo de un concepto biológico clave como es la noción de que las plantas son seres vivos es limitada en edades tempranas. Está conceptualización es precaria antes de los 8 años, sin embargo, el dominio sobre este concepto mejora paulatinamente con la edad. Cuanto más 
mayores son cada vez mejoran más en la categorización de las plantas como seres vivos y son más los elementos vitales dibujados en relación a las plantas, dándoles una mayor connotación de vida (agua, sol, lluvia, tierra, semillas...).

Se necesita investigar más en detalle estos aspectos, especialmente, intentando relacionar las producciones pictóricas y desarrollo del concepto de ser vivo por parte del alumnado. Junto a éstos, los estudios longitudinales e interculturales también podrían desempeñar un papel importante a la hora de examinar está relación. Además, un mayor análisis en las producciones pictóricas como puede ser el análisis del color y las formas geométricas pueden ayudar a contribuir en esta línea.

Con relación a las consecuencias educativas de la investigación presente, las conclusiones mostradas pueden ayudar a considerar el dibujo como un recurso útil en la evaluación del desarrollo conceptual en los primeros años de la infancia. La representación vegetal y los elementos pictóricos relacionados con estos (sol, fenómenos atmosféricos, insectos, agua...) parecen estar estrechamente unidos con el proceso de compresión de la noción de ser vivo. Esta idea está en la línea con estudios previos (Villarroel 2016; Villarroel e Infante 2012) que relacionan la producción pictórica y la comprensión conceptual, así como otras investigaciones que conexionan el pensamiento y la actividad pictórica en la infancia (Salmón y Lucas 2011).

Asimismo, un cambio metodológico hacia procedimiento educacionales más vinculados con las producciones pictóricas podrían ser una manera eficaz de direccionar la comprensión y construcción de lo que es el concepto de ser vivo. Aproximaciones racionales a la noción de ser vivo podrían no ser tan útiles en edades infantiles, por lo que se invita desde esta investigación a tratar otras vías alternativas, como en el caso de las plantas un acercamiento más relacionado con el dibujo.

\section{Agradecimientos}

Al autor le gustaría agradecer a los centros educativos Kueto Eskola y CEIP Albiz su participación y colaboración en la presente investigación. 


\section{Referencias Bibliográficas}

Anggoro, F. K., Waxman, S. R., y Medin, D. L. (2005). The effects of naming practices on children's understanding of living things. En B. Bara, L. Barsalou y M. Bucciarelli (Eds.), Proceedings of the Twenty-Seventh Annual Meeting of the Cognitive Science Society (pp. 139-144). Mahwah, NJ: Lawrence Erlbaum Associates.

Bowker, R. (2007). Children's perceptions and learning about tropical rainforests: An analysis of their drawings. Environmental Education Research, 13(1), 75-96.

Carey, S. (1985). Conceptual change in childhood. Cambridge: MIT Press.

Casler, K., y Kelemen, D. (2005). Young children's rapid learning about artifacts. Developmental Science, 8(6), 472-480.

Casler, K., y Kelemen, D. (2007). Reasoning about artifacts at 24 months: The developing teleo-functional stance. Cognition, 103(1), 120-130.

Cherney, I. D., Seiwert, C. S., Dickey, T. M., y Flichtbeil, J. D. (2006). Children's drawings: A mirror to their minds. Educational Psychology, 26(1), 127-142.

Dove, J. (2011). Rainforest depiction in children's resources. Journal of Biological Education, 45(4), 208-212.

Erickson, J. E., Keil, F. C., y Lockhart, K. L. (2010). Sensing the coherence of biology in contrast to psychology: Young children's use of causal relations to distinguish two foundational domains. Child Development, 81, 390-409.

Gatt, S., Tunnicliffe, S. D., Borg, K., y Lautier, K. (2007). Young maltese children's ideas about plants. Journal of Biological Education, 41(3), 117-122.

Gelman, S. A. (2003). The essential child: Origins of essentialism in everyday thought. New York: Oxford University Press.

Greif, M. L., Kemler Nelson, D. G., Keil, F. C., y Gutierrez,F. (2006). What do preschoolers want to know about animals and artifacts? Psychological Science, $17,455-459$.

Hannust, T., y Kikas, E. (2007). Children's knowledge of astronomy and its change in the course of learning. Early Childhood Research Quarterly, 22(1), 89-104.

Hatano, G., y Inagaki, K. (2002). Young children's thinking about biological world Nueva York: Psychology Press. 
Holliday, E. L., Harrison, L. J., y McLeod, S. (2009). Listening to children with communication impairment talking through their drawings. Journal of Early Childhood Research, 7(3), 244-263.

Inagaki, K., y Hatano, G. (2008). Conceptual change in naïve biology. En S. Vosniadou (Ed.), International Handbook of Research on Conceptual Change (pp. 240-262). Nueva York: Routledge.

Kelemen, D., y Rosset, E. (2009). The human function compunction: Teleological explanation in adults. Cognition, 111(1), 138-143.

Kemler Nelson, D. G., Egan, C. L., y Holt, M. B. (2001). When preschoolers ask, "what is it?' 'what do they want to know about artifacts. Psychological Science, 15, 384389.

Kline, R. B., y American Psychological Association. (2004). Beyond significance testing: Reforming data analysis methods in behavioral research. Washington: American Psychology Association.

Leddon, E. M., Waxman, S. R., y Medin, D. L. (2009). Unmasking “alive": Children's appreciation of a concept linking all living things. Journal of Cognition and Development, 9(4), 461-473.

Leddon, E. M., Waxman, S. R., y Medin, D. L. (2011). What does it mean to 'live' and 'die'? A cross-linguistic analysis of parent-child conversations in english and indonesian. British Journal of Developmental Psychology, 29(3), 375-395.

Malchiodi, C. A. (1998). Understanding children's drawings. Nueva York: Guilford Press.

Medin, D. L., y Atran, S. (2004). The native mind: Biological categorization and reasoning in development and across cultures. Psychological Review, 111, 960983.

Meunier, B., y Cordier, F. (2004). The plant category: When does it come to be organized? Enfance, 56, 163-185.

Michael, O., y Rajuan, M. (2009). Perceptions of 'the other' in children's drawings: An intercultural project among Bedouin and Jewish children. Journal of Peace Education, 6(1), 69-86.

Molina, M., Van de Walle, Gretchen A, Condry, K., y Spelke, E. S. (2004). The animateinanimate distinction in infancy: Developing sensitivity to constraints on human actions. Journal of Cognition and Development, 5(4), 399-426. 
Morse, D. T. (1999). MINSIZE2: A computer program for determining effect size and minimum sample size for statistical significance for univariate, multivariate, and nonparametric tests. Educational and Psychological Measurement, 59(3), 518531.

Opfer, J. E., y Siegler, R. S. (2004). Revisiting preschoolers' living things concept: A microgenetic analysis of conceptual change in basic biology. Cognitive Psychology, 49(4), 301-332.

Piaget, J. (1929). The child's conception ofthe world. London: Kegan Paul.

Prajapati, B., Dunne, M., y Armstrong, R. (2010). Sample size estimation and statistical power analyses. Optometry Today, 16(07), 10-18.

Prokop, P., y Fancovicová, J. (2006). Students' ideas about the human body: Do they really draw what they know. Journal of Baltic Science Education, 2(10), 86-95.

Rennie, L. J., y Jarvis, T. (1995). Children's choice of drawings to communicate their ideas about technology. Research in Science Education, 25(3), 239-252.

Richards, D. D., y Siegler, R. S. (1984). The effects of task requirements on children's life judgments. Child Development, 55, 1687-1696.

Rollins, J. A. (2005). Tell me about it: drawing as a communication tool for children with cancer. Journal of Pediatric Oncology Nursing, 22(4), 203-221.

Salmon, A. K., y Lucas, T. (2011). Exploring young children's conceptions about thinking. Journal of Research in Childhood Education, 25(4), 364-375.

Shepardson, D. P., Wee, B., Priddy, M., Schellenberger, L., y Harbor, J. (2007). What is a watershed? Implications of student conceptions for environmental science education and the National Science Education Standards. Science Education, 91(4), 554-578.

Siegel, S., y Castellan, N. (1988). Non-para-metric statistics for the behavioral sciences. Nueva York: McGraw-Hill.

Snaddon, J. L., Turner, E. C., y Foster, W. A. (2008). Children's perceptions of rainforest biodiversity: Which animals have the lion's share of environmental awareness? PLoS One, 3(7), e2579.

Solomon, G. E., y Zaitchik, D. (2012). Folkbiology. Wiley Interdisciplinary Reviews: Cognitive Science, 3(1), 105-115.

Spelke, E. S., y Kinzler, K. D. (2007). Core knowledge. Developmental Science, 10(1), 89-96. 
Topsakal, U. U., y Oversby, J. (2012). Turkish student teachers' ideas about diagrams of a flower and a plant cell. Journal of Biological Education, 46(2), 81-92.

Reiss, M. J., y Tunnicliffe, S. D. (2001). Students' understandings of human organs and organ systems. Research in Science Education, 31(3), 383-399.

Tunnicliffe, S. D. (2004). Where Does the Drink Go? Primary Science Review, 85, 8-10.

Villarroel, J. D. (2013). Environmental judgment in early childhood and its relationship with the understanding of the concept of living beings. Springer Plus, 2(87), 1-13. doi:10.1186/2193-1801-2-87

Villarroel, J. D. (2016). Young children's drawings of plant life: A study concerning the use of colours and its relationship with age. Journal of Biological Education, $50(1), 41-53$.

Villarroel, J. D., y Infante, G. (2014). Early understanding of the concept of living things: An examination of young children's drawings of plant life. Journal of Biological Education, 48(3), 119-126.

Waxman, S. (2005). Why is the concept "living thing'" so elusive? Concepts, languages, and the development of folk biology. In W. Ahn, R. L. Goldstone, B. C. Love, A. B. Markman, y P. Wolff (Eds.), Categorization inside and outside the laboratory: Essays in honor of Douglas L. Medin (pp. 49-67). Washington, DC: American Psychological Association.

Yorek, N., Sahin, M., y Aydin, H. (2009). Are animals 'more alive'than plants? animisticanthropocentric construction of life concept. Eurasia Journal of Mathematics, Science \& Technology Education, 5(4), 369-378. Wolschke-Bulmahn, J., y Gröning, G. (1994). Children's Comics: An Opportunity for Education to Know and to Care for Nature?. Children's Environments, 232-242. 